appear to converge, the projections of the Fourier Transforms of this divergent series onto $|\omega|<\Omega$ are converging both in the $L^{2}$ norm and the maximum norm.

\title{
BIBLIOGRAPHY
}

1. J. Douglas, Jr. and T. M. Gallie, An approximate solution of an improper boundary value problem, Duke Math. J. vol. 26 (1959) pp. 339-347.

2. F. John, Numerical solution of the equation of heat conduction for preceding times, Ann. Mat. Pura Appl. ser. IV vol. 40 (1955) pp. 129-142.

3. C. Pucci, Sui problemi di Cauchy non "ben posti," Atti Accad. Naz. Lincei. Rend. Cl. Sci. Fis. Mat. Nat. vol. 18 (1955) pp. 473-477.

International Business Machines Corporation

\section{PERRON-FROBENIUS THEORY AND THE ZEROS OF POLYNOMIALS}

\author{
HERBERT S. WILF ${ }^{1}$
}

1. Introduction. Our purpose here is to show that many of the classical root location theorems for polynomial equations, normally derived by the methods of complex analysis, can be obtained easily, and in a purely algebraic manner, from the Perron-Frobenius theorems on matrices with nonnegative elements. An important result of this approach is a "minimax" principle which gives precisely the largest root of an equation which dominates the given one. It will be seen that from this principle the above-mentioned location theorems follow, and can be sharpened almost at will. Finally some applications to the theory of orthogonal polynomials will be made, the result again being a minimax principle for the largest zero from which twosided bounds can be deduced by specialization.

2. Cauchy's Theorem. Let $C$ be an $n \times n$ complex matrix, and let $C^{+}$be given by

$$
\left(C^{+}\right)_{i j}=\left|C_{i j}\right|, \quad(i, j=1, \cdots, n) .
$$

A lemma of Wielandt [1] asserts that if $C^{+}$is irreducible, $\gamma$ is any eigenvalue of $C$, and $r$ is the largest real eigenvalue of $C^{+}$, then $|\gamma| \leqq r$.

Let

Presented to the Society, January 24, 1961 ; received by the editors May 13, 1960.

1 This work was supported in part by the National Science Foundation. 


$$
f(z)=a_{0}+a_{1} z+\cdots+a_{n} z^{n}
$$

where the $a_{j}$ are arbitrary complex numbers except that

$$
a_{0} a_{n} \neq 0 \text {. }
$$

The companion matrix of $f(z)$ is

$$
C=\left(\begin{array}{rrrrr}
-\frac{a_{n-1}}{a_{n}} & -\frac{a_{n-2}}{a_{n}} & - & \cdots & -\frac{a_{0}}{a_{n}} \\
1 & 0 & 0 & \cdots & 0 \\
\vdots & & & & \vdots \\
0 & 0 & \cdots & 1 & 0
\end{array}\right)
$$

while

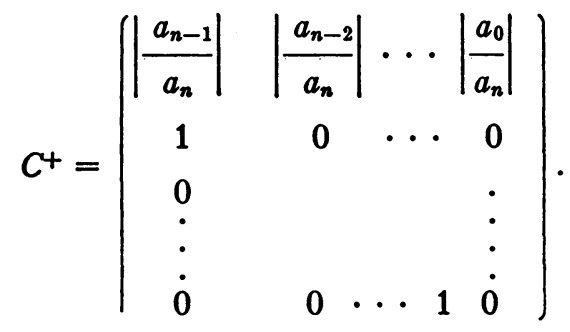

It is easy to see that (3) implies the irreducibility of (5). Hence all the eigenvalues of $C$ (zeros of $f(z))$ are dominated by the largest real eigenvalue of $C^{+}$, namely the real positive root of

(6) $\quad f_{c}(z)=-\left|a_{0}\right|-\left|a_{1}\right| z-\cdots-\left|a_{n-1}\right| z^{n-1}+\left|a_{n}\right| z^{n}$.

The result is due to Cauchy [2]. Applying the Perron-Frobenius theorem to the matrix $\mathrm{C}^{+}$yields the following information about the zeros of (6):

THEOREM 1. The polynomial $f_{c}(z)$ of (6) has a zero $r$ which is real, positive, simple, and which is not exceeded by the modulus of any other zero of $f_{c}(z)$. If precisely $h$ zeros of $f_{c}(z)$ have modulus $r$, then each of these satisfies

$$
z^{h}-r^{h}=0,
$$

and then the set of all zeros of $f_{c}(z)$ is carried into itself by a rotation of the complex plane through an angle $2 \pi / h$. Finally, the number $r$ is precisely given by

$$
r=\min _{x_{i} \geq 0} \max _{1 \leq i \leq n} \frac{\left(C^{+} x\right)_{i}}{x_{i}}
$$


Replacing $C^{+}$by its transpose in (8) and carrying out the multiplication indicated we deduce

THEOREM 2. The number

$$
r=\min _{x_{i} \geqq 0} \max _{1 \leqq i \leqq n}\left\{\left|\frac{a_{n-i}}{a_{n}}\right| \frac{x_{1}}{x_{i}}+\frac{x_{i+1}}{x_{i}}\right\}, \quad\left(x_{n+1}=0\right),
$$

is the real, positive, dominant root of (6), and hence if $z$ is a zero of $f(z)$ in (2), and $x_{1}, \cdots, x_{n}$ are arbitrarily chosen positive numbers, we have

$$
|z| \leqq \max _{1 \leqq i \leqq n}\left\{\left|\frac{a_{n-i}}{a_{n}}\right| \frac{x_{1}}{x_{i}}+\frac{x_{i+1}}{x_{i}}\right\}, \quad\left(x_{n+1}=0\right) .
$$

\section{Some applications.}

Taking $x_{i}=1(i=1, \cdots, n)$, we get

$$
|z| \leqq \max \left\{\left|\frac{a_{n-1}}{a_{n}}\right|+1,\left|\frac{a_{n-2}}{a_{n}}\right|+1, \cdots,\left|\frac{a_{1}}{a_{n}}\right|+1,\left|\frac{a_{0}}{a_{n}}\right|\right\}
$$

due to Cauchy [2].

With $x_{i}=\left|a_{n-i+1} / a_{n}\right| x_{1}(i=1, \cdots, n)$, there results

$$
|z| \leqq \max \left\{\left|\frac{a_{0}}{a_{1}}\right|, 2\left|\frac{a_{1}}{a_{2}}\right|, \cdots, 2\left|\frac{a_{n-1}}{a_{n}}\right|\right\}
$$

due to Kojima [2].

Next, with $x_{i}=\rho^{i}(i=1, \cdots, n ; \rho>0)$,

$$
|z| \leqq \max \left\{\left|\frac{a_{n-1}}{a_{n}}\right|+\rho,\left|\frac{a_{n-2}}{a_{n}}\right| \frac{1}{\rho}+\rho, \cdots,\left|\frac{a_{1}}{a_{n}}\right| \frac{1}{\rho^{n-2}}+\rho,\left|\frac{a_{0}}{a_{n}}\right| \frac{1}{\rho^{n-1}}\right\}
$$

$$
\leqq \rho+\max _{1 \leqq i \leqq n}\left\{\left|\frac{a_{n-i}}{a_{n}}\right| \rho^{-(i-1)}\right\},
$$

which also results from (11) by considering $f(z / \rho)$. If $\rho$ is chosen so as to make the two terms above equal, we find

$$
|z| \leqq 2 \max _{1 \leqq i \leqq n}\left|\frac{a_{n-i}}{a_{n}}\right|^{1 / i}
$$

due to Fujiwara [2]. It is easy to see, however, that better choices of $\rho$ can be made.

As a final application of the same idea, let $\left\{\phi_{n}(x)\right\}$ be a sequence of orthogonal polynomials on a real interval, normalized, and also standardized so that the leading coefficient $k_{n}$ of $\phi_{n}(x)$ is positive. 
Then

$$
x \phi_{n}(x)=B_{n} \phi_{n-1}(x)+B_{n+1} \phi_{n+1}(x)+A_{n} \phi_{n}(x)
$$

where

$$
B_{n}=k_{n-1} / k_{n}>0 \quad(n=1,2, \cdots) .
$$

It is well known that the zeros of $\phi_{n}(x)$ are the eigenvalues of the symmetric Jacobi matrix

$$
J=\left(\begin{array}{ccccc}
A_{0} & B_{1} & & \\
B_{1} & A_{1} & B_{2} & 0 \\
& B_{2} & A_{2} & \ldots & \\
& & \ddots & \ddots & \\
0 & & \ddots & \ddots & B_{n-1} \\
& & & B_{n-1} & A_{n-1}
\end{array}\right) .
$$

Hence if we have

$$
A_{n} \geqq 0, \quad(n=0,1, \cdots),
$$

the matrix $J$ is nonnegative and irreducible, and if $x_{n n}$ denotes the largest zero of $\phi_{n}(x)$ we find, as before,

$$
\begin{aligned}
x_{n n} & =\min _{\rho_{i} \geq 0} \max _{0 \leqq i \leqq n-1}\left\{B_{i} \rho_{i}+A_{i}+\frac{B_{i+1}}{\rho_{i+1}}\right\} \\
& =\max _{\rho_{i} \geq 0} \min _{0 \leqq i \leqq n-1}\left\{B_{i} \rho_{i}+A_{i}+\frac{B_{i+1}}{\rho_{i+1}}\right\}, \quad\left(\rho_{0}=1, \rho_{n}=0\right) .
\end{aligned}
$$

Again, various two-sided inequalities result from simple choices of the $\rho_{i}$, which in several familiar special cases correctly reproduce the first term of the asymptotically best inequalities. The upper bound given by (19) when all $\rho_{i}=1$ is a theorem of Shohat [3].

\section{REFERENCES}

1. F. R. Gantmacher, Applications of the theory of matrices, New York, Interscience Publishers, 1959.

2. M. Marden, The geometry of the zeros of polynomials in the complex plane, Mathematical Surveys, American Mathematical Society, 1949.

3. J. Shohat, The relation of the classical orthogonal polynomials to the polynomials of Appell, Amer. J. Math. vol. 58 (1936) pp. 453-464.

UNIVERSITY OF ILLINOIS 